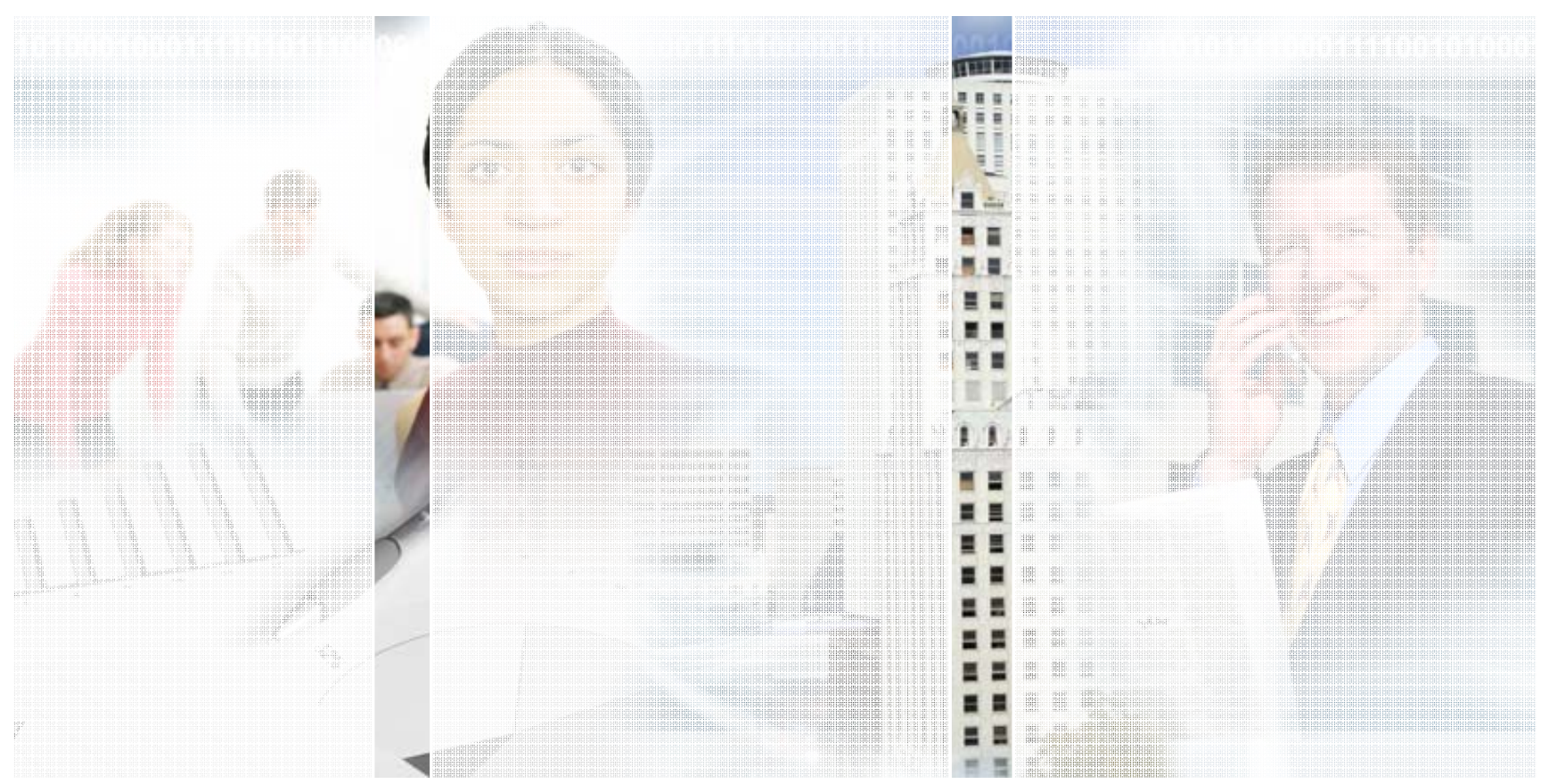

\title{
Create Centre of Excellence (CoE) for Better Business
}

(This article provides a perspective on COE from the relevance of IT Industry and business which is ideally seen in today's competitive world) 


\section{What is CoE?}

In today's world, the Centre of Excellence (COE) applies to any organization who wants to create and use excellent state of art and showcase its technological, service and business oriented capabilities to the entire world in a competitive environment. CoE is the combined effect of emerging business model and technologies.

Centre of Excellence (COE) refers to creation of assets for an organization and reaping maximum business benefits from the usage of such assets. The objective of creating CoE for any organization is always customer centric and is meant for providing unique solutions to their customers.

The Centers of Excellence (COE) trend very recently has gained traction within the IT department of large organizations. In fact, the META Group refers to the model as "the next step in IT evolution" (Source: The Application Center of Excellence, META Group, 2003).

IT organizations of all types and dimensions are embracing the CoE model as a practical way of consistent and continuous ally improvement of their business operations. Industry analysts also support the move to the COE model.

A CoE is an organization, focused on optimizing application or service characteristics such as quality, performance, or availability. It provides management an automation platform for processes, consulting, and support services, leadership as well as advocacy to optimize these attributes.

In IT world, CoE acts as a central point of contact to facilitate collaboration between the lines of business, enterprise architects, database administrators and application developers. It helps in speed adoption, improve knowledge sharing, lower the Total Cost of Ownership (TOC), and broaden the reach and context of enterprise application deployment, migrations, consolidations and upgrades.

CoE Brings together a dedicated team of appropriate domain expertise and enables the organizations to identify core patterns of integration, share best practices, leverage skills and standard tools across the enterprise.

A CoE provides a central source of standardized products $\&$ services with expertise, and best practices. It can also provide the entire organization with visibility into quality and performance parameters of the delivered application and support functions, helping them to keep everyone informed and keeping the applications and services aligned with business objectives.

The strategy and roadmap for $\mathrm{CoE}$ are required to launch and sustain the business needs. Without a COE, you will lack the necessary ecosystem and opportunity to optimize resources, funding, competencies, and governance structure, hence the ideology of the CoE should be in synchronization with the philosophy of the organization business imperative.

\section{Why CoE is required?}

Creating a CoE is a proven, yet flexible approach to obtain the strategic benefits of business in all its forms, while maintaining control on costs, quality and operation. CoE can bring tremendous benefits to an organization, by creating the necessary state of art to provide service and product excellence to their valued customers and users. CoE is used to provide the best technical solutions to the challenging, complex, and evolving business needs of customers.

There are various reasons for organizations to create $\mathrm{COE}$ and the most common are as follows:

- To keep pace with technologies and their impact on how business is conducted, Organizations create dedicated Centers of Excellence $(\mathrm{CoE})$ in various technologies. 
- Creating a CoE addresses the need of organizations to maximize the usage of hardware, software and highly skilled personnel to provide solutions to the customers, partners or end users.

- A CoE enables to centralize technology as well as create a repository of solutions, and reusable components, sharing best practices and knowledge base. Reuse of technology in some cases can be leveraged across different segments of business.

- It provides timely information, measures, KPI's to the users and senior management throughout the organization enabling them to take appropriate and timely decisions in competitive advantage.

- If COE is created for providing services and products then enterprises can rapidly realize the benefits of a COE by leveraging service and product expertise with the help of consistent methodology and industry knowledge.

- CoE for some organizations is meant for Implementing the most optimal technologies, architectures, and frameworks to provide best-of-breed solutions and services to the customers and Clients.

- Some organizations use CoE to enable implementation of all needed regulatory requirements. requirements, resources, or starting point for building a CoE; however, the flexibility of the CoE model enables company's to start with smaller scale, using existing resources, and achieve tangible benefits almost immediately.

Organizations use various methods to set-up and manage $\mathrm{CoE}$; considering $\mathrm{CoE}$ have components of PMO or considering centralized or decentralized structure. The CoE model can also be a critical asset for the distributed organizations, providing centralized processes, infrastructure and, and reporting. COE is primarily supposed to be a combination of soft and hard infrastructure based solution centre.

One of the key advantages in setting-up of the CoE is that it can be built with minimal incremental expenditure and service-product mix. You can evolve and scale up its resources, services, and capabilities progressively as its value is delivered to the business.

The interesting part of $\mathrm{CoE}$ is that the value of the CoE is not limited to the IT Department. Expertise, tools, repository and best practices relevant to the product, services and technology enables quality, performance, and availability of such assets for every business function throughout the organization including partners, suppliers, vendors, and even customer organizations.

\section{Setting up of CoE}

\section{Objectives of setting-up of CoE}

Setting-up of CoE

requires a coordinated effort across the dimensions of people, process, technology and management's continuous support and commitment to convert CoE as a business solution centre or asset.

Two organsiastion may not have the same

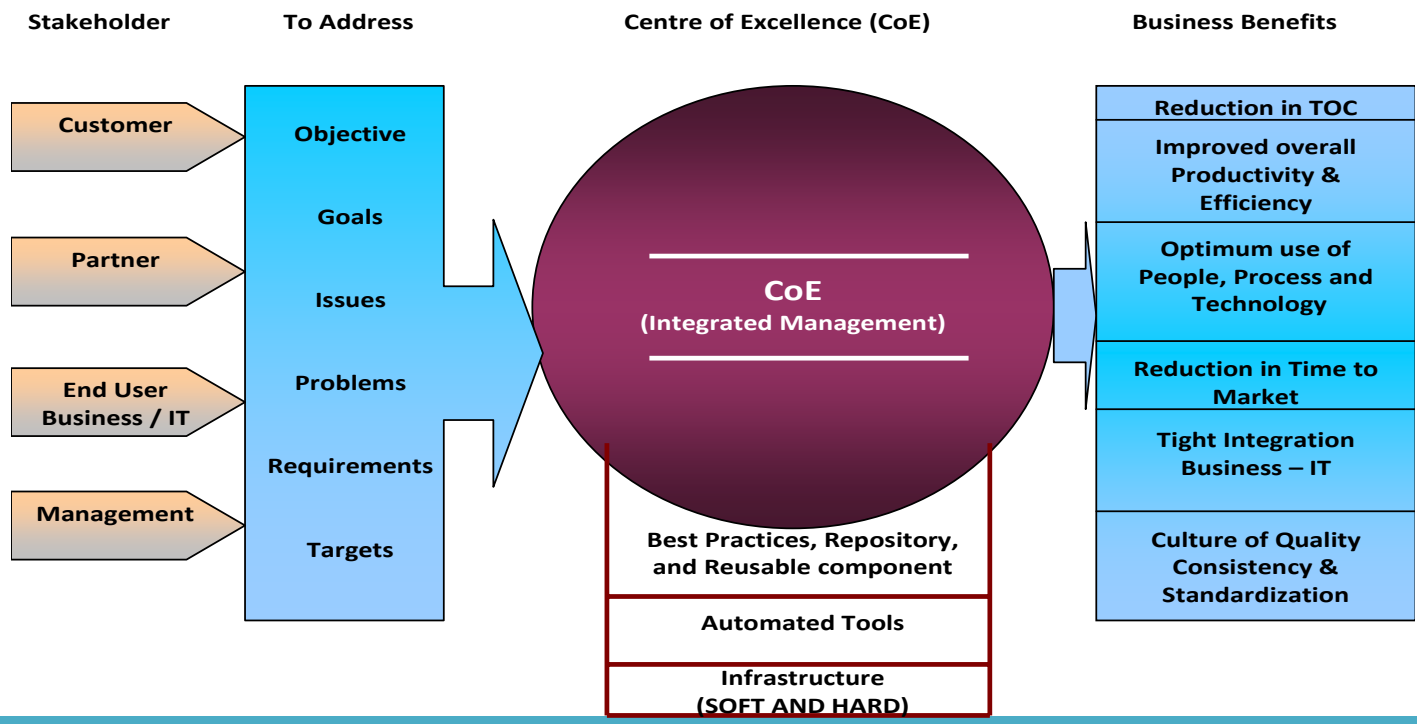


Organizations have to set clear goals while creating COE to protect the investments and fulfill the business objectives. Typically following are the common examples of setting goals for $\mathrm{CoE}$ :

- Catalyze the growth and improve the competitiveness in industry

- Create a unique platform for creating global solutions

- Implement the vision of providing a single place where users can access all services, information, reports, KPIs, scorecards and other metrics essential for their business requirements. Create a standard vehicle for deployment of best practices

- Develop and apply research, innovative technologies for development / maintenance and support services

- Integration of services such as operational support, application management, enhancements, Infrastructure management and change management, address multisite, multi location requirements and reduce Total Cost of Ownership (TCO)

- Establish norms for an organization and enhance user productivity. Promote and ensure that the reusable-assets are produced. Optimize the use of all products and services implemented in a costeffective manner, contributing to the overall Company's success.

- Provide customers a more comprehensive, reliable, and adaptable hardware and software solutions in order to speed their time-to-market

- Establish CoE to provide support to business and in meeting organizations global or domestic IT implementation requirements.

- Development of strong competencies in technology, enhance the value of the application to provide standardized solution and better integration. Improved retention of key personnel and service levels and be an "Encyclopedia" for solutions.
- Delivery of strategic business value through technical excellence, process excellence and leveraging economies of scale

\section{COE's Basic needs}

CoE needs to determine on scope and boundaries while centralizing or decentralizing their targeted activities across several projects, teams, applications, services, technologies, business units, or the entire enterprise.

A good start for setting-up CoE is, always to do a readiness assessment for establishing $\mathrm{COE}$ in the form interviews or by circulating a questionnaire to key stakeholders of the organization; to understand the need and objectives of establishing CoE. The score of critical parameters with weighted average will provide some clues on how you would evolve CoE model. Such assessments shall provide considerations for people, process, product and services before taking the next step forward.

People: The analysis of existing and needed skills to run CoE requires assessment; the skills which are common and can compliment similar development and support functions may need separate listing. The areas where there is need of knowledge transfer; have to be identified for CoE. In some cases, there may be a need to upgrade existing knowledge or even to bring in totally new skill sets. Ensuring smooth, efficient knowledge transfer between employees and among cross-Line of Business (LOB) project teams is critical for making a good start with CoE. Further, utilizing outside experts, SME's for organizational design, training, and mentoring may be required.

Organization setting-up CoE have to create a strong role based structure with projected resources and how CoE will handle need of business in an integrated fashion by optimum use of its infrastructure, technology and tools 
Process: When you apply industry best practices, you can create world-class processes. Ensuring consistent processes built from a high level of expertise while delivering services or product from CoE will result in more of them completing successfully in less time for less money. At the same time, a flexible approach to adapt to the new framework, standards and quality models will improve quality of delivery through $\mathrm{COE}$ and organizational cultures too.

Infrastructure: Robust infrastructure and automation platforms with usable tools are essential for the success of the CoE. One of the primary goals the COE shall work to eliminate piecemeal tools and incompatible or absolute platforms among all IT applications.

By standardizing on industry-leading, state-ofthe-art technologies, organizations can accelerate time-to-market, improve speed, accuracy and realize significant tangible savings. Standardized CoE oriented technologies can also help your organization maintain superior efficiency of delivered services; automate processes to ensure consistency and repeatability; and gain control and visibility into CoE activities.

Products \& Services: The CoE should collect and package any best practices relevant to the services or products it provides both internally and externally. Before you embark upon new service level requirements, it is important to assess current level of service commitments and what level of services would be required to be rendered which may provide improved value to the business. Often organization start with most simple business services or applications to gain some experience before they move on addressing critical ones

CoE offers integrated software, services, and best practices to help companies make the move to the CoE model quickly, efficiently, and cost-effectively. It is also used to optimize application and service quality, performance, availability, and problem resolution, while managing IT projects, their costs, and compliance. CoE Optimization Centers enable IT functional teams to work in a centralized, automated fashion - saving time, cutting costs, and increasing the effectiveness of critical IT activities. In addition, CoE Managed Services provides you with a pre-installed and managed central platform together with mentoring and coaching services supporting your move to the CoE model.

\section{Planning CoE}

Setting up of CoE requires careful considerations on infrastructure planning, operations, business facing IT, application development and support requirements. Executive sponsors have to plan, strategize and implement CoE.

Planning CoE shall have some primary key characteristics such as:

- It shall be business-led value proposition; business plan which can sustain for at least next 5 years Designed according to the enterprise size $\&$ needs and supported by latest technology in IT.

- $\quad$ Consider possibility of virtual organization; flexible to operate in central or distributed environment.

- $\quad$ Decide services and products or mix required to serve the business and the time frame?

- $\quad$ Optimization of current system usage (business and IT levels) shall be given considerations.

- Scope (development/ enhancement/ support) and complexity of the solution?

- $\quad$ Resource pool, full time, flexible, part time and type of resources (technical/ functional/ management/ support)?

- $\quad$ Skills and knowledge required for the staff to perform required activities in $\mathrm{COE}$.

- $\quad$ Training needs especially in the area of latest technology, practices and management 
- $\quad$ Capacity, availability of product or service needed to design and the overall agreed scope of responsibilities for the COE.

- Number of users and systems to be supported, under CoE umbrella, the existence or absence of local/ onsite support.

- $\quad$ The proposed governance model, regulatory and other compliance requirements to be fulfilled.

\section{Building an efficient CoE}

It is important to understand that there are no universally accepted standards for building COE's, as it will vary from organization to organization. Accordingly, there is no off-the-shelf solution or silver bullet for implementing a COE within an organization.

Before discussing an approach for implementing a COE it is important to understand some of the characteristics and objectives of a CoE (as stated above) and then specify rules of engagement to ensure its success.

While establishing a COE function, the key concept is to start with minimum operational requirements; it doesn't have to be perfect on Day one. It will evolve, grow and get matured as per the business needs. Overall, flexibility and agility to adapt to change, end user, business, and organizations requirements is critical for the $\mathrm{CoE}$ to drive value to the organization, and is paramount for the CoE's long term success.

The CoE should ideally be headed by a very senior IT person with good project, business and support experience who could influence and provide vision. The best CoE's have strong business representation both from a competency management perspective and delivery perspective. CoE will not deliver optimal value to the organization if IT functions and business work in silo

\section{How to Choose an Implementation Path}

Planning the implementation of CoE isn't a small task. Because there is organizational change involved, there are many aspects which need to be considered. Fortunately, you can take an incremental approach for implementing CoE.

Companies that are successful in implementing a CoE have not only focused on current problems that need to be resolved but also on the long term business benefits such as, reduced cost or improved time-to-market. The implementation of CoE includes maj or activities such as:

- Finding a champion to drive the CoE with proven ability to deliver.

- Communicating to management the longterm vision and value of the COE

- Finding the appropriate funding model

- Manage organizational changes and communicate the right message to stakeholders

- Building new skill sets

- Selecting, configuring or reusing/ augmenting hard and soft infrastructure and setting-up of environment.

- Creation of re-usable security integration, application and service level integration components

- Creation of strong governance and operational model

- Development of a system-level management dashboard, reporting and alert tracking mechanism

- Creation of a capacity planning and charge back systems

- Implementing industry standards, quality models and best practices. Documentation and control of major processes and procedures supported by your business rules, Integration points, and configuration and change control. 


\section{Managing CoE}

The CoE environment must be managed properly and optimized in order to demonstrate business value to the organization. Consider that working with $\mathrm{COE}$ must be more than managing services and products for the organization. It should represent holistic set of services and intellectual capital for the business.

As the results of ongoing management activities and by validating the successes or pit falls, CoE shall use the created environment to further improve the quality of delivery and measure the objectives set earlier by the organization.

CoE Management shall ensure that following aspects are addressed on the ongoing basis

- Aligning business owners with IT staff on a continual basis to arrive at common goal

- Identifying value-added and non-valueadded processes and practices, optimizing them from time to time

- Benchmarking results against initial ROI projections

- Planning for future enhancements and operational excellence and enhancing infrastructure, standards and procedures suitable to the new business processes or service requirements

- Committing and providing ongoing training and knowledge transfer to end users and customers on processes and procedures to be followed

- Institutionalizing change management

- Preservance of best practices, repository and reusable components

- Use CoE to address critical business problems.

- Monitor and report the KPIs, service level measures created by CoE to senior management and measure the success and value of the CoE against the metrics established during the planning phase.
- Implementation of quality models such as ISO9001, Six Sigma, and CMMI to improve maturity of process and practices with in CoE

\section{Business benefits of CoE}

- The key question for many CIOs today is not whether the CoE approach would benefit the organization, but rather how best to make the transition to the $\mathrm{COE}$ model from existing state. Those who have followed disciplined approach towards setting -up and implementing CoE successfully have realized many business benefits. CoE offers the means to coordinate the effort by creating a common set of resources, models and tools to meet the broad set of business needswhile taking advantage of existing organization expertise, standards and infrastructure.

- Creating a CoE allows companies to decrease and optimize technology costs, increase organizational efficiency due to shared resources; more quickly comply with reporting regulations, and improve overall business agility.

- The CoE enables organizations to set up and operate centralized technologies and utilize effectively a repository of seamless business knowledge and best practices, sharing of which reduces the learning curve for the team members and reduction in "Time-To-Production" as learning is shared across the projects.

- CoE provides a common implementation model, where by reducing the time to respond during the business issues. It also improves the productivity of teams across the enterprise.

- Application development/service products, staff are integrated and made conveniently accessible to all CoE teams through one source, so there is no need to replicate 
expensive resources. You can expect sizable reduction in total headcount

- Tight Integration: The CoE model can help organizations synchronize business goals with IT priorities, resulting in better business outcome. By defining, measuring and acting on KPIs, CoE helps keep the product and services aligned tightly with business needs

- Career advancement: The CoE model creates a compelling new career opportunity for IT professionals, helping the organization recruit and retain top talent.

- Consistency: Standardizing quality best practices helps ensure consistent, costeffective, and rapid implementation of optimized quality processes.

- Practicality: Building a Quality CoE is an achievable goal. You can start on a small scale, leveraging existing resources, and expand its capabilities as the value is proven.

- Automation and appropriate management control of CoE lowers the cost of ownership and minimizes the disruptions to business while improving quality of delivery and business responsiveness resulting in competitive advantages

- Decreased time to market: The rapid response capability of the CoE helps reduce total time for development and servicing, resulting in faster time to market and on-time response to business needs - all at a lower total cost.

- Culture of quality: The transition from islands of expertise to standardized quality processes and toolsets helps focus the organization on quality issues and speeds the evolution to a culture of quality.

\section{Conclusion}

Think of the CoE as the holder of the keys to the business success. Mature organizations have mature practices and engage which needs constant improvement. Your road to a fully functional, wellmanaged, cost-efficient, and highly regarded COE shall lead to an industry leadership in business technology optimization (BTO).

The CoE model has already proven its value in hundreds of IT Organizations worldwide. The COE is an efficient change agent, driving optimization in the organization.

Individual initiatives and attempts to achieve better quality in delivery typically have a very limited visibility, scope and impact as an organization; moreover there are often major roadblocks to implementing them. Deploying and evolving a CoE enables organizations to improve quality and performance of its product and service delivery in an organized way, at their own pace, while simultaneously cutting costs. It provides greater visibility by measuring its outcome and comparing the same with its business goals.

CoE provides economies of scale along with reduction in Total Cost of Ownership (TOC). It provides maximum realization of an integration investment, made by an organization.

Organizations have shown year-over year average value of more than 15- $20 \%$ of improvement in their business outcome by adapting to COE model. 


\section{References}

- Realizing business value with Testing Centre of Excellence-Manoj Narayan and Somil Katiyar (Infosys)

- Application Delivery: Centre of Excellence Evolution-white paper from Mercurywww. mercury. com

- Deploying a Centre of Excellence for data integration by IBM corp.

- Building TIBCO- Centre of Excellence Thought to Realization whitepaper from HCL Technologies

- $\quad$ SAP- Centre of Excellence-By Bob White my SAP Support blog

- Building and Managing a Quality Centre of Excellence

- Your Path To A Test Center Of Excellence by Carey Schwaber with Marc Cecere, J acqueline Stone, and Katie SmillieForrester Research, Inc

\section{About the Author}

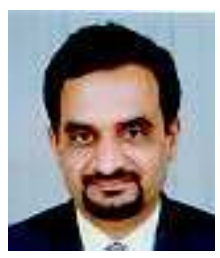

The author Sunil V Tadwalkar is Principal Consultant in Quality Consulting Group (QPRIME) of Satyam Computer Services Ltd. He has over 24 years of experience in IT and Mfg organizations His expertise lies in process consulting and project/program management, outsourcing, knowledge management, competency building, strategic initiatives and Quality Function Management. He may be contacted on:

Sunil Tadwalkar@Satyam.com Contact: +91-20-

30534231 (M): +91-9850992146 